\title{
ZUR POLLENANALYTISCHEN DIAGNOSE SÜDOSTEUROPÄISCHER HONIGE
}

\author{
F. RUT'TER \\ Institut für Bienenkunde, Oberursel/Taunus
}

ZUSAMMENFASSUNG .

Importhonige aus Ungarn sind meist hell und spät kandierend. Mikroskopisch findet sich eine charakteristische Kombination von Robinia- und Onobrychis-Pollen mit Körnern einer xerothermen Flora (Cerinthe minor, Stachys anmuus, Loranthus europaeus, Helianthemum, Verbascum, Zea mais, Cucumis). Honige aus dem östlichsten Randbezirk Osterreichs, in dem dieselbe Pflanzengesellschaft vorkommt (s. Abb.) lassen sich pollenanalytisch von diesen Importhonigen nicht unterscheiden. Leichter gelingt dies bei österreichischen Robinia-Onobrychis Honigen aus weiter westlich gelegenen Gebieten mit einer artenärmeren Begleitflora mitteleuropäischer Prägung.

Importhonige aus Jugoslawien sind meist Honigtau-Castanea-Honige. Eine charakteristische Pollenform in diesen Honigen ist Loranthus europaeus. In Osterreich werden Honigtau-Castanea. Honige vor allem in der Weststeiermark geerntet; hier fehlt aber Loranthus. Diese sehr auffällige Pollenform bildet also ein wertvolles Hilfsmittel zur Bestimmung der geographischen Herkunft dieser Honige.

Die pollenanalytische Charakterisierung von Honigen aus SO-Europa ist ein Problem von erheblicher praktischer Bedeutung. Denn alle Länder Südosteuropas erzielen eine hohe, relativ konstante Honigproduktion und alle Honigimportländer Mitteleuropas beziehen alljährlich beträchtliche Honigmengen aus diesen Gebieten. Diese Honige sind in ihrer geschmacklichen Qualität den mitteleuropäischen Konsumenten meist vertrauter als manche andere Importhonige und uiberdies auch deshalb attraktiv, weil sie aus handelspolitischen Gründen zu sehr niedrigen Preisen angeboten werden - zu viel niedrigeren, als im Herkunftsland selbst.

MaURIZIo (I959) ist es gelungen, bei der Untersuchung einer grösseren Serie jugoslawischer Honige eine Charakteristik " südosteuropäischer Honige " herauszuarbeiten. Sie weist dabei auf die zu überwindenden Schwierigkeiten hin, da ähnliche Typen auch in gewissen Gebieten Mittel - und Westeuropas geerntet werden, und spricht von einer "Feindiagnose des geschulten Pollenanalytikers ". 
In Österreich sind nun diese Schwierigkeiten noch ungleich grösser. Denn der pannonische Florenbereich mit kontinentalem Klima reicht über die Ostgrenze Österreichs herein bis an den Ostrand der Alpen und bis in die "mährische Senke " nördlich von Wien. In diesem Gebiet werden mit grosser Regelmässigkeit gute Honigernten erzielt - in manchen Jahren die einzigen in ganz Österreich. Diese Honige, die sich nach Konsistenz und Geschmack nicht von gewissen ungarischen, rumänischen oder jugoslawischen Importhonigen unterscheiden lassen, werden in Wien und in den westlichen Bundesländern zum Verkauf angeboten, wo sie immer wieder Anlass zu Beanstandungen geben. Die pollenanalytische Beurteilung solcher Honige hat sich demnach vor allem mit zwei Problemen auseinanderzusetzen :

I. Beanstandungen von Honigen aus dem Osten Österreichs durch Konsumenten oder Imker, weil diese Honige in Geschmack und Konsistenz (Robinia-Honige kandieren bekanntlich nicht oder sehr spät) von den lokal geernteten abweichen. Diese Gruppe stellt den Hauptanteil der eingesandten Proben.

2. Falsche Deklaration eines Importhonigs.

Die grosse Masse der aus Suidosteuropa exportierten Honige lässt sich auf Grund ihrer pflanzlichen Herkunft in zwei Gruppen gliedern :

\section{A. Pannonische Robinia-Onobrychis-Honige}

Es sind dies sehr helle, meist erst sehr spät kandierende Honige, die zum Grossteil aus Ungarn stammen.

B. Honigtau-Castanea-Honige aus Jugoslawien von ziemlich dunkler Färbung.

\section{Pannonische Robinia-Onobrychis-Honige}

Im Pollenpräparat dieser Honige findet man Robinia- und Onobrychis-Körner in wechselnder Häufigkeit und in wechselndem Verhältnis. Durchaus nicht immer ist eine der beiden Formen in der Häufigkeitsstufe des Leitpollens zu finden, aber nur selten wird eine gänzlich fehlen. In der Mehrzahl der Fälle handelt es sich um Mischhonige mit überaus formenreichen Pollenspektrum. Die oben gewählte Benennung soll also nicht einen Sortenhonig im Sinne der Pollenanalyse bezeichnen, sondern einen ganz bestimmten Honigtyp, der vor allem durch eine sehr spezifische Begleitflora charakterisiert wird.

An sich ist weder der Pollen von Onobrychis noch der von Robinia ein brauchbares Indiz für die geographische Herkunft eines Honigs, auch dann nicht, wenn er als Leitpollen vorkommt. Onobrychis-Honige werden nach ZANDER (I937) und MAURizIo (I958) ausser in SO-Europa auch in Frankreich, Spanien, Luxemburg, der Westschweiz und in Süddeutschland gefunden. Honige mit Robinia-Pollen als Leit- oder Begleitpollen sind nach denselben Autoren auch aus Italien, dem Tessin und der Rheinpfalz bekannt.

Sehr viel seltener ist schon die Kombination Robinia-Onobrychis. Maurizıo (r949, I958) fand diese Zusammenstellung häufig in Honigen aus Osterreich, aber nur je ein Mal in solchen aus der Schweiz (Tessin), Jugoslawien und Luxemburg (ungarische Honige wurden damals von ihr nicht untersucht). Das Vorkommen von Robinia- und Onobrychis-Pollen zusammen ist also offenbar recht typisch für die ungarische Tiefebene und die Ausläufer ihrer Flora im östlichen Grenzbezirk Österreichs, es erlaubt aber noch keineswegs eine eindeutige Charakterisierung. 
Lavandula vera

Lavandula stoechas

Lavandula dentata

Lavandula latifolia

Cistus albidus

Cistus monspeliensis

Cistus crispus

Cistus salviaefolius

Cistus laurifolius

Cistus varius

Cistus Mumbyi

Cistus ladaniferus

Cistus hirsutus

Cistus heferophyllus

Helion themum sp.

Calluno vulgaris

Erica vagons

Erica umbeliata

Erica cinereo

Hypecoum sp.

Rosmarinus $s p$

Sarothamnus type

Centaurea cyanus

Thymus sp.

Citrus $s$

Olea sp.

Onobrychis sp.

Sinapis sp.

Rubus sp.

Echium sp

Taraxacum . sp.

Lotus sp.

Trifolium repens

Zea Moys

Rosaceae

Vicıa sp.

Erica arborea

Vitis Sp.

Solix sp.

Amygdalus sp.

Melilotus sp.

Castanea vulgaris

Eucalyptus sp.

Hedera helix

Compositae type Onopordum Compositae type Serratula

Plantogo sp.

Mentho sp.

Rumex acetosella

Tragopogon

Ombelliferoceoe

Trifolium pratense

Campanulacece

Centaureo jaceo

Proteaceae

Tilio sp

Pinus sp.

Robinia pseudocacio

Buxus sempervirens

Rhododendron ferrugineum

Cornus sanguinea

Lonicera $\mathrm{sp}$.

Arbutus unedo

vaccinum sp.

Cucurbitaceo

Brassica napus

Chenopodicceoe

Fogopyrum esculentum

Helianthus sp.

Papaver sp.

Corex sp

Dipsocaceae

Lavondin

Malvacece

Fumana sp

Stochys sp.

Liliacede

Spireo sp.

Compositoe type Aster

Caryophyllaceae

Rophanus raphanistrum

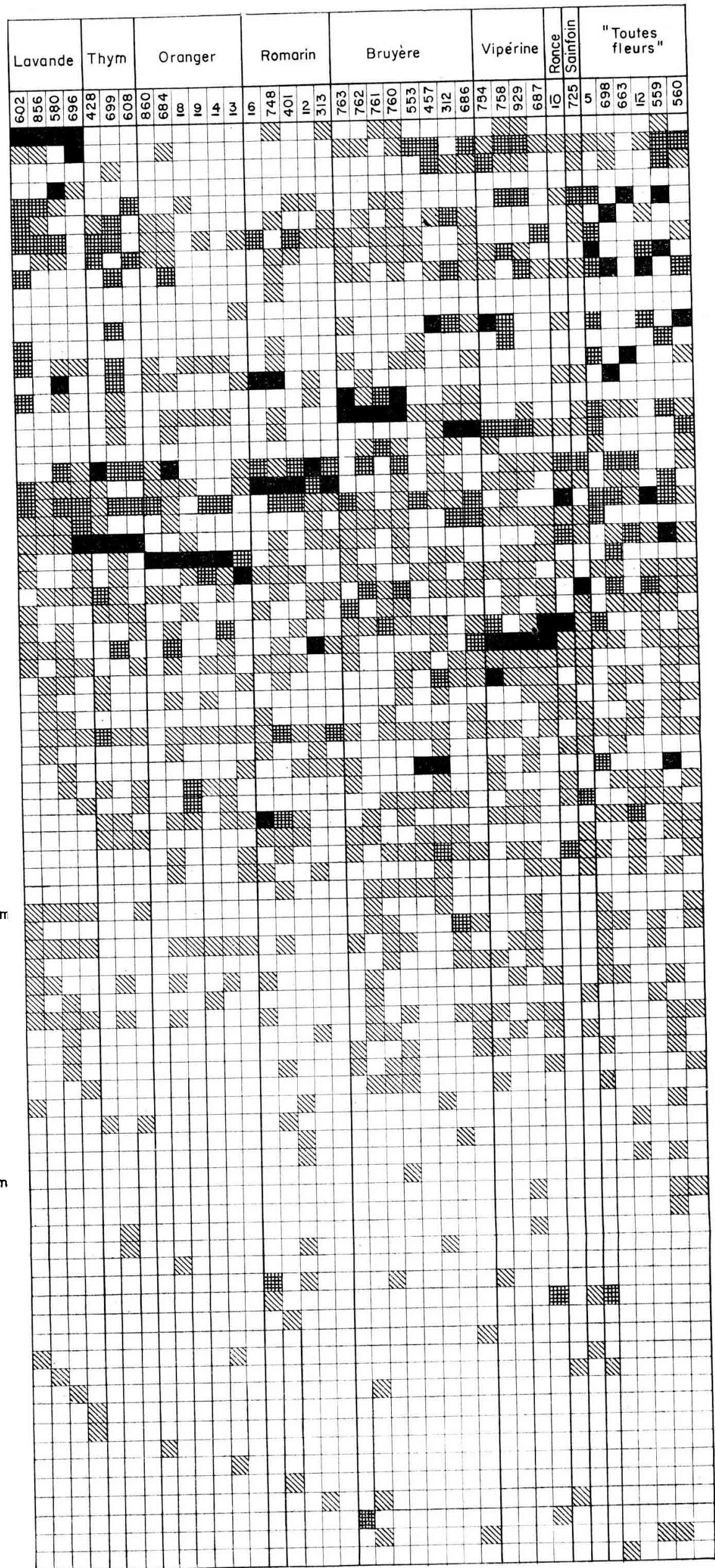

Fic: 1. .... Spedre pollinique des trente huil mels éludiés

en noir : pollens dominants

en hachures doubles : pollens d'accompagnement

en hachures simples : pollens isolés

(Voir explications complementaires au chipitre "matéricl et métho.es») 
Eine solche ist erst möglich auf Grund einer Analyse der Begleitflora, wie sie von HAzsidnsky (1952) durchgeführt wurde. Diese Begleitflora ist auffällig vor allem durch ihren ausserordentlichen Artenreichtum. Wie bei alpinen Honigen zählt man unter Ioo Pollenkörnern 25-30 verschiedene Spezies-Typen. Das zweite Charakteristikum dieser Honige ist das Vorkommen von Pollenkörnern einer ganzen Anzahl ausgesprochen xerothermer Elemente.

Das Pollenspektrum pannonischer Robinia-Onobyvchis-Honige lässt sich regelmässig in dtei Sektoren zerlegen :

a. Uncharakteristische Formen, besonders der Feldflora. Hier sind vor allem zu nennen die immer zahlreichen Cruciferen (vor allem Raphanus und Sinapis), Centaurea cyanus, Vicia, Quercus. Lediglich bei den ebenfalls regelmässig anzutreffenden Trifolium-Arten ist eine Besonderheit hervorzuheben: Neben Trifolium pratense und T. repens findet sich in zahlreichen Honigen Pollen von T. incarnatum eine Form, die sonst in Mitteleuropa zu den Seltenheiten gehört.

b. Xero- und thermophile Elemente aus Ruderal-und Trockenrasen-Associationen und von Kulturplanzen, die aber nicht auf den pontopannonischen Florenbereich beschränkt sind. Hierher gehören die schon oben erwähnten Onobrychis und Robinia. Ferner Verbascum, Hypericum, Helianthemum, Echium, Papaver und verschiєdene Chenopodiaceen. Von Kulturpflanzen sind zu erwähnen Zea, Cucumis, Vitis und Fagopyrum.

c. Typische Formen des ponto-pannonischen Florenbereiches, von denen vor allem drei für die Diagnose wichtig sind:

Stachys anmums, ein Ackerunkraut, das bis vor einigen Jahren im August die abgeernteten Getreidefelder mit seinen weissgelblichen Bliiten bedeckte und vielfach eine sehr beachtliche Späternte lieferte. Heute ist diese Massenvegetation infolge der modernen Bewirtschaftungsmethoden zwar verschwunden, aber als "Indikator" ist das Pollenkorn von Stachys anmus (neben dem ron anderen Labiaten des Lamium-Typs) in den meisten pannonischen Honigen zu findet. Es gelangt auch in die Frühsommerhonige, dx ein Teil der Pflanzen nicht erst in Juli-August, sondern schon im Juni - zwischen den Getreidehalmen — zur Blüte gelangt.

Cerinthe minor ist nach Hegi (I925) ein pontisches Element, das verstreut zwar auch weiter westlich (bis Obetbayern und Franken) gefunden wird, dort aber als Archaeophyt nur an Ruderal- und Segetalstandorten vorkommt. HAzsinsky (I962) hat das Pollenkorn in etwa Io p. Ioo der von ihm untersuchten ungarischen Robimia-Honigen als Begleit- und Einzelpollen gefunden; für jugoslawische Honige wird es von MaURIzIO (r959) nicht erwähnt. In Österreich ist es in typischen, pannonischen Robinia-Onobrychis-Honigen relativ häufig anzutreffen (im nördlichen Burgenland in 63 p. Ioo der untersuchten Honige, am Sïdwestrand des Wiener Beckens in I5 p. roo). Meist tritt es als Einzelpollen auf, in zwei Fällen aber sogar als Leitpollen. In anderen Gebieten Österreichs wurde dieses Pollenkorn bisher nicht gefunden - ausser überraschenderweise in einzelnen sehr trockenen inneralpinen Tälern, worüber in anderem Zusammenhang zu berichten sein wird.

Loranthus europaeus, die Eichenmistel aus Südosteuropa und Kleinasien, kommt im Osten Österreichs als Parasit vorwiegend auf Quercus Robur vor. Das sehr auffälig gestaltete Pollenkorn bildet nach Maurizio (I959) eine Charakterform jugoslawischer Castanea-Waldhonige. In Österreich wird es in Honigen des pannonischen 
Bereiches überall dort gefunden, wo grössere Baumbestände vorhanden sind (mittleres Burgenland, Stadtgebiet ron Wien, "Weinriertel "zwischen Wien und der Grenze der CSSR; RvTTMis (IgGI). Nie die Häutigkeitsgrenze des Einzelpollens überschreitend, ist es in Österreich in Robinia-()nobrychis-Honigen noch weiter westlich $z u$ finden als $z$. B. Cerinhe. I’ür Ungarn wird es ron Hazslinszky (I952) nicht erwähnt. es ist aber auch dort im bewaldeten Hügelland mit Sicherheit zu erwarten.

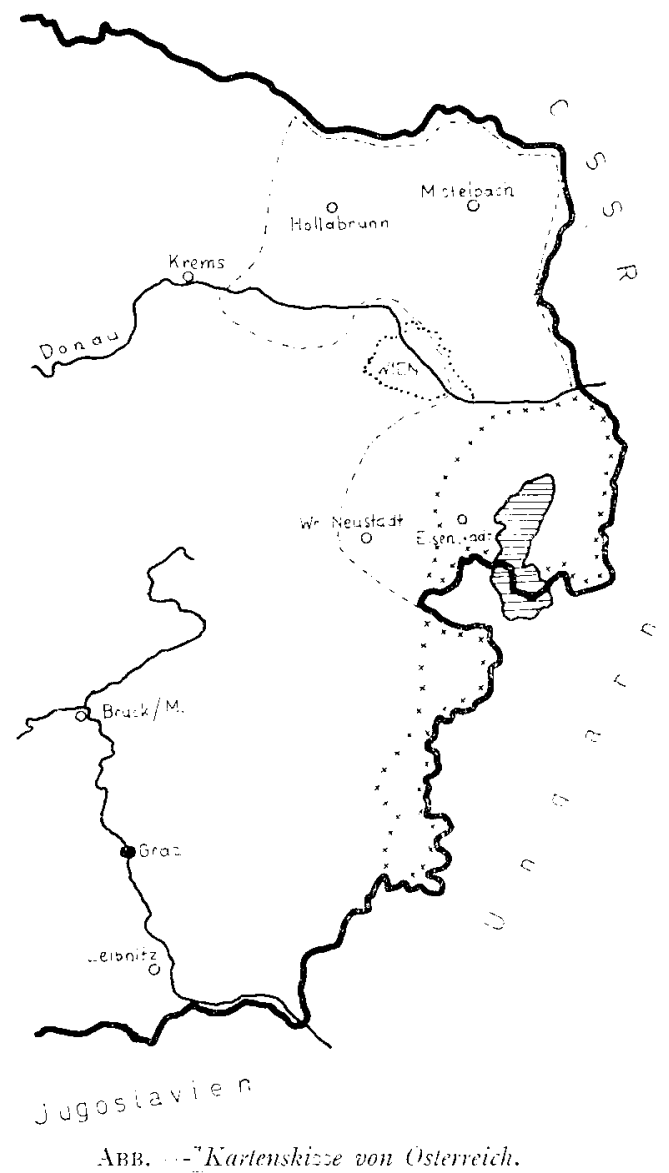

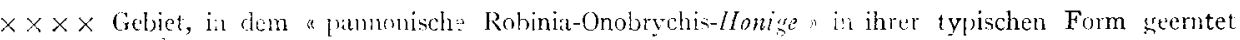
werden

Westarenze des Gebietes, in dem häulisr Robinia-Onobrychis-Honige festzustellen sind.

Neben der Aufzählung der Bestandteile einer sehr charakteristischen Pflanzengesellschaft, welche mehr oder weniger ausgeprägt das Bild der sommerheissen und trockenen pannonischen Ebene liefert, ist es nicht minder wichtig, das zu erwähnen, was in diesen Honigen nicht vorkommt. Es fehlen den typischen Robinia-OnobrychisHonigen des pannonischen Bereiches vor allem die Bewohner feuchter Wiesen und Wälder, die in den Frülısommerhonigen Mitteleuropas regelmässig anzutreffen sind : Taraxacum, Leucanthemum, Lychnis, Mrosotis, Cmbelliferen A, Rubus usw. 
Lirst an der schon fenchteren Westgrenze dar Rolinia-Onolwychis-Honige finden wir eine Zone, in der die pannonische Begleitflora durch die oben erwähnten feuchtigkeitsliebenden lilemente ersetzt wird. An wenigsten weit nach Westen dringen ausgesprochen xerophile Formen wie Helianthemun und Cerinthe ror. Hand in Hand mit dem Verschwinden der pannonischen tilemente geht eine Verringerung der Artenzali.

\section{Jusoslawische Honiglan-Castanea-Honige}

I)ie jugoslawischen Honige können in einige gut definierte 'ly'pen aufgegliedert

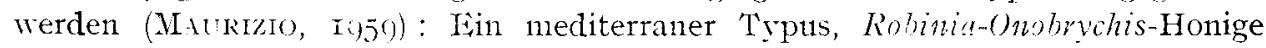
ähnlich den oben beschriebenen, Tilia- und Fagopynum-Honige. Weitaus an häufigsten fand MAURIzIo aber eine Vergesellschaftung ron Honigtau-Bestandteilen mit Castanta-Pollen (als I,eit- oder Begleitpollen) und einer sehr artenreichen Begleitflora (I,eguminosen mit Onolonchis, Robinia, Trifolium incannalum, Labiaten M und $s$, Compositen, Craciferen). Als besonders charakteristisch heht MArRizin das Vorkommen der Pollenkörner von Loranthus europaens und Comabis sativa hervor.

In Vergleich "ul den oben beschriebonen Honigen der pannonischen Fbene lassen dicie Honigo auf ein wesentlich feuchteres Klima schliessen. Ausgesprochen xerophile Flemente treten stark in den Hintergrund. Durch einen Vergleich der durchschnittlichen Niedarschlagsmenge ron Mistelbach (nördlich ron Wien) und Marburg (Marihor, Slomenien) lässt sich diese Vermutung bestätigen : Durch:ichnittlicher Jahresniederschiag ron Wistelbach $450 \mathrm{~mm}$, von Marburg $890 \mathrm{~mm}$.

I) Kombination von Castane:-pollen und Honigtnu wird in einigen ästlichen Iandesteilen Österreichs keineswegs selten gefunden, ror allem in der Weststeiermark, im mittleren Burgenland und in Wiencrwald. 1)en Schlissel zur loifferentialdiagnose gegeniber jugoslawischen Wald-Castanea-Honigen liefert das sehr leicht kenutliche Loranthus-Korn. In Osterreicin gehört Lowanthus nicht zum Bestandteil von Castanea-Honigen. Winzig in einem eng begrenzten (iebiet des mittleren Burgenlandes nahe der ungarischen Grenze (Iockenhans) wnde bisher diese Kombination Honigtau-Castanca-Loranthus getunden.

\section{DISK USSION}

Der sorgfältig durclgeführten Analyse ron Honigen aus dem Osten Österreichs mit dem Ziel, zu einem Kriterium für die Lnterscheidung gegenüher Importhonigen aus südosteuropa zu gelangen, war also nur ein lialber Frfolg beschieden. Jugoslawische Importhonige können in allgemeinen rasch und relativ sicher als solche erkannt werden: Wurde das Vorliegen eines Wald-Castanea-Honigs diagnostiziert, so wird das ganze Präparat bei schwacher Vergrösserung nach Loranthus-Pollen durchstucht. Wird ein solcher gefunden, so handelt es sich mit sehr grosser Wahrscheinlichkeit um einen jugoslawichen Honig.

Schwieriger liegen die Dinge bei Rohinia-Onobrychis-Honigen. Zeigt ein solcher Honig in seinem Pollengehalt die typischen Filemente der pannonischen Flora, so kann zwischen einer Herkunft aus Ungarn oder Österreich nicht entschieden werden. 
Denn dieselbe Flora, wie sie in den rauptproduktionsgebieten des "Ungarischen Honigs» vorhanden ist, erstreckt sich nach Westen bis gegen Wien. Nur wenn sich in einem Honig Robinia und Onobrychis mit der artenärmeren mitteleuropäischen Wiesenflora vergesellschaftet findet, kann auf eine österreichische Herkunft, meist aus dem Hauptproduktionsgebiet dieses Honigs, dem westlichen Weinviertel, geschlossen werden.

Die Nachbarschaft dieses Gebietes zum pannonischen Florenbereich zeigt sich in dem regelmässigen Auftreten einzelner Pollenkörner östlichen von Formen (Stachys annuus, Loranthus europaeus o. a.).

\title{
RÉSUMÉ
}

DIAGNOSTIC POLLINIQUE DES MELS DU SUD-EST DE L'EUROPE

Presque tous les miels importés de Hongrie sont clairs et cristallisent lentement. L'examen microscopique révèle une combinaison caractéristique des pollens de Robinia et Onobrychis avec les grains d'une flore xérophile (Cerinthe minor, Stachys annuus, Loranthus europeaus, Helianthemum, Verbascum, Zea mais, Cucumis). Les miels venant de la bordure orientale de l'Autriche, oì se présente la même association végétale, ne se distinguent pas, par l'analyse pollinique, de ces miels d'importation. Il est plus facile de distinguer les miels autrichiens de Robinia-Onobrychis, originaires de région situées plus à l'ouest, qui ont une flore d'accompagnement plus pauvre en espèces, de type européen du centre.

La plupart des miels importés de Yougoslavie proviennent d'une miellée de Castanea et de miellat. Lne forme caractéristique des pollens de ces miels est constituée par Loranthus europaeus. En Autriche les miels de miellat et de Castanea sont récoltés principalement en Styrie de l'ouest où ne se trouve pas Loranthus. Ces formes très reconnaissables de pollen constituent donc un moyen précieux pour la détermination de l'origine géographique de ces miels.

\section{SUMMARY}

\author{
POLLEN ANALYSIS OF TIE HONEYS OF SOUTH-EASTERN LUROPE
}

Nearly all the honeys imported from Hungary are clear, and crystallise slowly. Microscopic examination reveals a characteristic combination of the pollens of Robinia and Onobrychis with the grains of a xerophylous flora (Cerinthe minor, Stachys annuus, Hellianthemum, Verbascum, Zea mais, Cucumis. The honeys of the eastern parts of Austria, where the same association of plants is found, is not distinguishable, by pollen analysis, from these imported honeys. It is easier to distinguish the Austrian honeys from Robinia-Onobrychis, which originate from more westerly regions, which have a much less varied flora, of the central European type.

The majority of honeys imported from Yugoslavia derive from a Castanea honeydew. A characteristic form of the pollens of these honeys is constituted by Loranthus europaeus. In Austria, honeydew and Castanea honeys are principally harvested in western Styria where Loranthus is not found. These easily recognisable forms of pollen thus constitute an important means of determining the geographic origine of these honeys.

\section{LITERATUR}

Hazslinszky B., 1952. Qualitative und quantitative Untersuchung ungarischer Robinienhonige. Mitt. Ungar. Akad. Wiss. Biol. Sekt., 1, (3), 3 I 7-4I7 (ungarisch mit deutcher und russicher Zusammenfassung). HEGI G., 1925. Illustrierte Flora von Mitteleuropa, Bd. V/3, 2190-219r. Pichler, Wien. 
Maurizio A., 1949. Pollenanalytische Untersuchungen an Honig und Pollenhöschen. Beihefte zur Schie eiz. Bienen-Zeitung, 2, (I8), 320-42 I.

Maurizio A., 1958. Beitrag zur quantitativen Pollenanalyse des Honigs. 3. - Absoluter Gehalt pflanzlicher Bestandteile in Esparsette, Luzerne, Orangen und Rapshonigen. Ann. Abeille, 2, 93-106.

Matrizio A., 1959. Das mikroskopische Bild jugoslawischer Importhonige. Z. Bienenforsch., 5, 8-22.

Ruttner F., ig6r. Der Pollen der Eichenmistel (Loranthus europaeus JAco.) als Charaliterform in üsterreichischen Honigen. Z. Bienenforsch., 5, 220-226.

ZANDER E., 1937. Beilräge zur Herkunflsbestimmung bei Honig, II. Leipzig, I937. 